\title{
False Information Diffusion in Public Crisis on Interpersonal Network and Intervention Strategies
}

\author{
Zhu Xiaoxia, Hao Jiajia* and Hu Haiju \\ ( Economics Management School, Yanshan University 066004 Qinhuangdao, \\ Hebei Province, China) \\ zhuxiaoxia@ysu.edu.cn
}

\begin{abstract}
With the development of economy, the public crisis has been increasing frequently and false information took shape because of the public's assentation and lack of recognition. The diffusion of false information has intensified the public panic and anxiety. Beside, it may cause secondary damage easily. Therefore, the management department of false information in public crisis information should control the diffusion of the false information quickly in order to reduce the loss. By the diffusion of the network of false information in public crisis based on the scale-free network (BA), this paper defines the nature of each social individual from the perspective of multi-agent. On the basis of these properties, this paper defines the individual opinion interaction rules when communicating. The research concludes that active individuals of information diffusion on interpersonal network are not the people with a great amount of friends, but the ones who have few friends. Nevertheless, the management department of false information in public crisis should maintain a circuitous neutral attitude rather than the "all-or nothing" coercive measures when intervene the diffusion process of the false information diffusion. Besides, the influencing social individuals are the "opinion leaders" who have a wide range of interpersonal communication.
\end{abstract}

Keywords: public crisis; false information; interpersonal network; Scale-free networks; Multi-body simulation

\section{Introduction}

With the rapid economic development, a number of social stability matters have been intensifying, such as population, resources, environment, efficiency and fair. Social environment and economic environment will become more complex and difficult to predict. Therefore, the public crisis will be more and more abundant. However, people tend to express their views on the situation when public crisis occur. Therefore, "false information" comes into being because of the inevitable differences between information expression and real information in the process of information communication.

Those false information lacking of objective descriptions of situation, such as rumors, messages or gossips are bound to increase public panic and anxiety. The diffusion false information in public crisis plays a more and more important role in the evolution process, increasing the complexity of the public crisis and uncertainty, and intensifying the damaging of public crisis. Besides, it acts as a hindrance to the crisis restore, even endanger the social and political stability. Studies show that in the diffusion process of false information, the final influences and the measures of most people are often decided by the interpersonal relationship ${ }^{[1]}$.

To control the diffusion range and speed of false information effectively as well as decreasing the secondary information dissemination harm when the crisis occurs, this

* Corresponding Author 
paper studies the dynamic mechanism of the diffusion of false information from the perspective of interpersonal relationships. This study will find out the diffusion rule of false information in public crisis by using the method of multi-body simulation and the visualization of the diffusion false information in public crisis. In addition, the intervene of the diffusion false information in public crisis helps the appear of the best intervention strategies and intervention plans to provide reference for the public crisis management department to manage false information.

\section{Literature Review}

From the existing research, the spreads of false information in public crisis such as rumors are similar. Therefore, most of the existing spread models of rumor take example by the infectious disease model. Rapoport and some other researchers are the earliest users of the spread of infectious disease model to research information problems ${ }^{[2]}$; Goffman and Newill explain the final state of the spread of rumors employing the stability of the infectious disease model ${ }^{[3]}$; Daley and Kendall put forward the spread of rumors mathematical model in $1960 \mathrm{~s}$, and the population is divided into several model states, the basic status including: S (Susceptible) - tingible state; I (Infected) --infection status; R (Recovered) - the removed or immune/recovery state ${ }^{[4]}$. According to different population state named by employing rumors spread model: SIR model (tingible groups were infected, and then back to health and immunity), SIS model (tingible group after infection, returned to the tingible state). Then some scholars put forward some other models such as SIRS ${ }^{[5]}$ and SIRH ${ }^{[6]}$ combining with the complexity of network transmission. Most rumors spread models of the population are divided into several categories according to rumor spreading effect, and assume that the probability of two kinds of state conversion to obey certain mathematical distribution. Since then, Maki D, Thomson M and Murray I D have employed mathematical model to study the rumors, and these studies mainly focused on the theoretical analysis ${ }^{[7-8]}$.

In addition, with the deepening of the dynamics research in recent years, the spread of false information transmission based on the dynamics have focused more attention. Some researchers like Dickinson makes analysis of the spread of infectious diseases compared with rumors spread mechanism, and summarize the evolution process both in the communication process and the related time ${ }^{[9]}$. Other researchers like Thompson set up the Daley-Kendall model similar to rumor model, considering the effect of diversity of population in the rumor spread. They found that people activity is the most sensitive parameters that influence the spread of rumors, and increasing activity can control the size of the rumor spread ${ }^{[10]}$. Kawachi has considered different population acceptance of rumors, and finite and infinite dimensional dynamic model are established to determine whether the rumor spreads or not ${ }^{[11]}$. Besides, Seki with other researchers have considered the influence of different contact for final spread, and the spread of the mathematical simulation method was used to study the final results ${ }^{[12]}$. In addition, Huo with some others research the utility model between the spread of rumors and the interaction of the government's emergency, using the data simulation to draw the phase diagram of the system $^{[13]}$. On the basis of these researches, they put forward the strategies and policy to make recommendations about emergency management. Other scholars like Zhong Qi, Qi Wei and Zhang Ke divided information into true information and false information, making analysis of the process of crisis information diffusion from the perspective of ecology. Based on the Lotka-Volterra model, this research constructs the social crisis information diffusion model and makes simulation and empirical analysis of the problem of the spread of social crisis information. It is found that the crisis information diffusion is closely related to the substitute coefficient and diffusion rate ${ }^{[14]}$.

By employing the theory of small world network, Zanette established rumors spread model and analyzed the influence of each factor to the spread of rumor ${ }^{[15]}$. The theory of 
complex adaptive system (CAS) modeling method makes system members as adaptable individuals. Each subject can interacts and makes autonomous learning between individuals and individuals, or between individuals and the environment, changing behaviors according to the experience of learning ${ }^{[16-19]}$. Complex system modeling method can explain simple rumor spreading process. However, it limited to simple interaction rules and the subjects also have similar characteristics. Besides, the reaction process between different subjects reflecting the real social interactions also exist some insignificance.

From the existing researches, although the rumors based on mathematical model are highly abstract, rigorous logic and of universality application, it is intuitive to the process description. Rumors spread close to display can be expressed in mathematical model, but can not be solved. In addition, infectious disease of the differential dynamic equation was established according to the data statistics of macroscopic mathematical model. Dynamic model for analysis of the micro transmission mechanism as well as cellular automata simulation method not consider the complexity of the social network of people. Therefore, these models assume that individuals in the society are homogeneous and fully mixed, and it is difficult to describe the degree of interaction between different factors like character, age, education and income. The network of the spread false information in public crisis is a typical complex networks together with a group of individuals in a certain system of special relationship. Based on this research, this study makes definition of diffusion network information interaction rules between different characteristics of the subject, and builds a complex network based on agent more false information transmission model. It makes visual clarity the diffusion process of false information through the netlogo multi-agent simulation platform and discusses the various interventions to control the spread of false information process. Therefore, it provides necessary theoretical guidance for the selection of intervention related to the false information in all kinds of public crisis, reducing the secondary hazards of false public crisis information diffusion.

\section{The Interpersonal Network Establishment of Public Crisis and False Information}

\subsection{The Scale-free Network Feature of Public Crisis and False Information Spreading}

The false information of public crisis has strong social attribute, when the false information of public crisis come out, more general public will pay attention to the information, that is, if the false information not be intervened, the amount of people who believe the false information in interpersonal network will increased. In the interpersonal network, because public education, culture, personality, age and other factors determine the different scope of their communicative ability and communication range. The individual are different, the demand of interpersonal communication are different. When people communicate with each other, the feature and number they resonate are different. As the same time, the root cause of the social life of individuals can communicate is share with others, it means, successful communication will establish the meaning which both sides can understand. If both of the individual can communicate normally, then they at least have topics or hobbies they can share. Therefore it is common that you can make friends with your original friend's friends, that is "Birds of a feather flock together". Most people can resonate with a limited number of friend, very few people can have a lot of friends who can be resonated with.

The feature that interpersonal network of false information spreading is the network continues to increase the number of individuals over time, the individual differs, the number and feature of friends they make are different, as well, the possibility to make 
friends with his original friends is larger, it has scale-free feature. According to the network's characteristic, the network has features as follows:

(1) Initialize a network containing $N$ nodes, increase the number of nodes in turn, it can meet the increasing requirement of individuals in the interpersonal network.

(2) To meet the requirement that individuals have different quantities of friends, and very few people have a lot of friends, most people have general amount of friends, we let the newly added node generates with other nodes to make edges by the following manner:

$p_{i}=\frac{k_{i}}{\sum_{j \in \Gamma} k_{j}}$

$\mathrm{K}$ stands for the node degree in the network, namely the amount of edges that node can make. The formula shows, if there are many lines, newly added node will have a greater probability of generating connection. The node degree generated by the formula obeys the power law, among the, very few node have more edges, most node have very few edges.

To meet the highly-collected feature of interpersonal network, according to the process of Figure 1, when the newly added node $\mathrm{H}$ establish contact with node $\mathrm{F}$ by certain probability, we choose one node from node F's neighbor node D, E, I randomly to establish contact, the process is shown by red, yellow and green dotted lines in Figure 1.

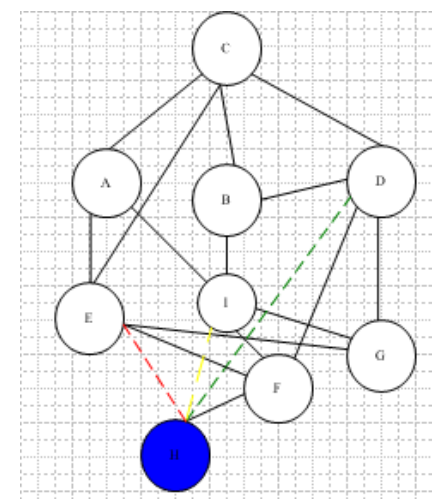

Figure 1. The Process of Adding Cluster Coefficient

\subsection{The Net Feature of Public Crisis and False Information Spreading}

(1) The individual (node) feature of public crisis and false information spreading

In society, different people accept different environment and education, therefore, the people they meet and opinions they have are all different. This paper makes some assumptions as follows:

(1) Individual opinions. When public crisis happen, people have original perspective toward the news without any surrounding interference. With the development of the news, people get positive or negative influence by public media and other individuals in the interpersonal network, so their opinions may get changed to varying degrees. Therefore people's attitude toward things is not static, and their attitude is not absolutely agreement or disagreement, people's attitude is always between them. Some correlation studies suppose that the individual opinions can be displayed by discrete value. For instance, 0 stands for disagreement, 1 stands for agreement. However, in view of instability of individual opinions, this paper uses continuous value to express individual opinions in the interpersonal network. Assume that individual opinions distribute randomly in the section $[0,1], 0$ stands for opposition, 1 stands for support, then the value in the section stands for 
opposition or support in varying degrees. Individual opinion $O_{i}$ changes inordinately with the time changing.

(2) Influence. Individuals in the interpersonal network have different impact to the surrounding neighbors due to their distinct status、age、 education background. Usually the node in alpha status has higher influence to his neighbors, so the impact is in direct proportion to the node value. This paper value $\inf l_{i}=\frac{k_{i}}{k_{\max }}, k_{i}$ stands for the degree of node $\mathrm{i}, k_{\max }$ stands for the max value in all the nodes of the network. ${ }^{\text {inf } l_{i}}$ can range in $[0,1]$.

(3) Susceptibility. When the crisis happens, besides influenced by the powerful people, whether the individual accept false information or not at last depends on the characteristics themselves. For example, a determined man will hardly be influenced by the surrounding, well the individuals who just spread news but never discriminate the fact or fiction will easily be influences by the surroundings. This paper chooses ${ }^{s u s_{i}}$ to stand for the degree of being influence. Assuming that the degree of being influence value randomly in the section of $[0,1], 0$ means they can hardly be influenced, 1 means they can easily be influenced. The value in the section stands for different degrees of susceptibility.

(4) In social life, every one communicate with a lot of people, but the number of people who can change his opinions is limited. So this paper excludes the ones who only has communication but can not change his opinions. Therefore, this paper supposes the node in the network can make six edges at most.

(2) The connection (edge) feature of public crisis and false information spreading

In society, there are closed and distant relationships among people, so the credibility among people is different. People who have close relationship usually have more credibility, and the normal friends have lower credibility. To describe the complex relationship of interpersonal network comprehensively, this paper bring in credibility variable ${ }^{\text {trust }_{i j}}$, and this variable trust $_{i j}$ is expressed by weight of edges, that is to say, $20 \%$ of the edges equals to weight of $0.8,80 \%$ of the edges equals to weight of 0.2. 0.8 means the credibility is higher, when the information is spreading, they may easily get influenced, while 0.2 means the credibility is lower, the impact is lower too.

\subsection{The Interaction Rules of Public Crisis and False Information Diffusion}

The interaction rules of public crisis and false information spreading is the dynamic mechanism during the false information spreading process. That means if the nodes get influenced by other nodes is the power to go on spreading false information.

At first, the presupposition of individuals exchanging their opinions is their opinion can not be too different. When the gap is over some threshold value, the resistance when they interact among the individuals is higher, only when the gap is less than a certain value, that is, when their opinions have certain similarity, the interact may happen.

$o_{i}(t+I)=O_{i}(t)+\frac{\sum_{\left|o_{i}(t)-o_{j}(t)\right|<\varphi} a_{i j} \times\left(o_{i}(t)-o_{j}(t)\right) \times \inf l_{j} \times \text { sus }_{i} \times \text { trust }_{i j}}{\sum_{\left|o_{i}(t)-o_{j}(t)\right|<\varphi} a_{i j}}$ 
In the formula, I stands for simulation step, ${ }^{a_{i j}}$ stands for all the weight of edges that collected with edge $i$. The variation of Node $i$ at $t$ moment is decided by the opinion gap between node $i$ and node $j$, the impact of node $j$, the susceptibility and the product of credibility of node $i$ and credibility of node $j$, at the same time, get the average value of all the node which meet the threshold value collected with node $i$.

\section{The Intervention Programs of Public Crisis and False Information Interpersonal Network}

\subsection{Intervention Simulation Program}

This paper focuses on intervention efforts and the impact of intervention strategy on the diffusion of public crisis and false information interpersonal network. The nature of information published by public media reflects intervention efforts from the government. The government intervene social individual with different nature denotes government intervention strategy.

Table 1. The Intervention Programs of Public Crisis and False Information Diffusion

\begin{tabular}{lllll}
\hline No intervention & $\begin{array}{l}\text { Confrontatio } \\
\text { nal } \\
\text { intervention } \\
\text { strategy }\end{array}$ & $\begin{array}{l}\text { Neutrality } \\
\text { intervention } \\
\text { strategy }\end{array}$ & $\begin{array}{l}\text { Supportive } \\
\text { intervention } \\
\text { strategy }\end{array}$ \\
intervention. & & & & \\
strategies & $A_{1}$ & $A_{2}$ & $A_{3}$ & $A_{4}$ \\
\hline $\begin{array}{l}\text { Point intervention } \\
\text { Multi-line } \\
\text { intervention }\end{array}$ & $B_{1}$ & $B_{2}$ & $B_{3}$ & $B_{4}$ \\
Radiant intervention & $C_{1}$ & $C_{2}$ & $C_{3}$ & $C_{4}$ \\
\hline
\end{tabular}

We explain the intervention programs of public crisis and false information in aspect of intervention efforts: (1) No intervention means free diffusion process of public crisis and false information; (2) Confrontational intervention strategy means when public crisis false information happened, public media on behalf of government absolutely against when they refute the rumor. In the simulation experiment, it is the note that its opinions increase value is 0 . (3) Neutrality intervention strategy means when public crisis false information happened, public media on behalf of government represents neutrality. In the simulation experiment, it is the note that its opinions increase value is 0.5. (4) Supportive intervention strategy means public media show supportive attitude. In the simulation experiment, it is the note that its opinions increase value is 1 .

We explain the intervention programs of public crisis and false information in aspect of intervention strategies: (1) Point intervention means when public crisis false information happened, the individuals who have small communication range accept the advice from public media. In the simulation experiment, it is the note that has only one line to select, its value is 1. (2) Multi-line intervention means we make the individuals whose communication range is in the middle accept the advice from public media. In the simulation experiment, it is the note which has three line to select, its value is 3. (3) Radiant intervention means we take the individuals who have the biggest communication 
range to accept the advice from public media. In the simulation experiment, it is the note which has six lines to select, its value is 6 .

From what has been discussed above, there are 12 intervention simulation programs:

$$
A_{1}, A_{2}, A_{3}, A_{4}, B_{1}, B_{2}, B_{3}, B_{4}, C_{1}, C_{2}, C_{3}, C_{4} .
$$

\subsection{Simulation Experiment Design}

From the point of intervention efforts, $A_{1} B_{1} C_{1}$ are simulation experiments without intervention program, which means that false information diffuse freely. We design the experiment as follows in Figure 2:

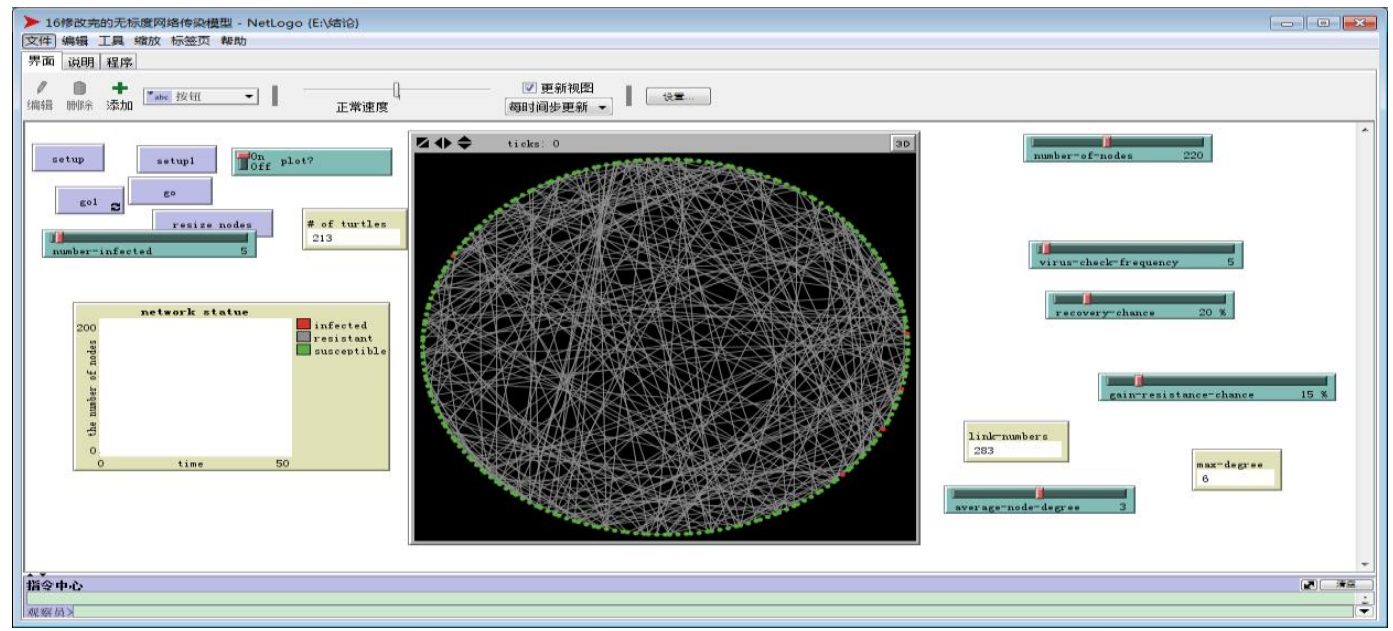

Figure 2. Experiment Design of False Information Diffuse Freely

(1) The Setup and go buttons build a scale-free network with different edge weights. The number-of-nodes decide initial number of nodes. The go routine will delete the notes which have six lines or more. Simultaneously, the final number of nodes will count the last nodes.

(2) Setup1 routine will setup the quantity and nature of note from the initial support information content. The quantity of notes with supportive attitude is controlled by the slider named number-infected. And the value of notes with initial supportive attitude will show by routine, which is the line number a note has.

(3) Data monitor include link-numbers, max-node-degree to make sure the network nodes connected to edges up to 6 , average-node-degree.

(4) Network statue draw, draw three states: infected (information recipients), susceptible (easy to receive information, but not), resistant (immune, someone who is not interested in the information, stop the spread). Graph of the number of individuals.

\subsection{Analysis of the Results from the Experiment that Public Crisis False Information Diffuse Freely}

When we execute $A_{1}, B_{1}, C_{1}$, we set the initially infected nodes value 1,3 and 6 by the routine go1 in Figure 3, 4, 5.

When public crisis false information occur and diffuse freely, the quantity of susceptible node decreased rapidly. The infected node increase rapidly. After reaching the largest outbreak scale, Fluctuation within a small range and stabilized. When the time is 
large enough, the quantity of infected node and susceptible node tends to 0 .The note return to immune status. It means that if we do nothing when public crisis false information occurs, the public crisis false information will disappear. The public will no longer spread false information.

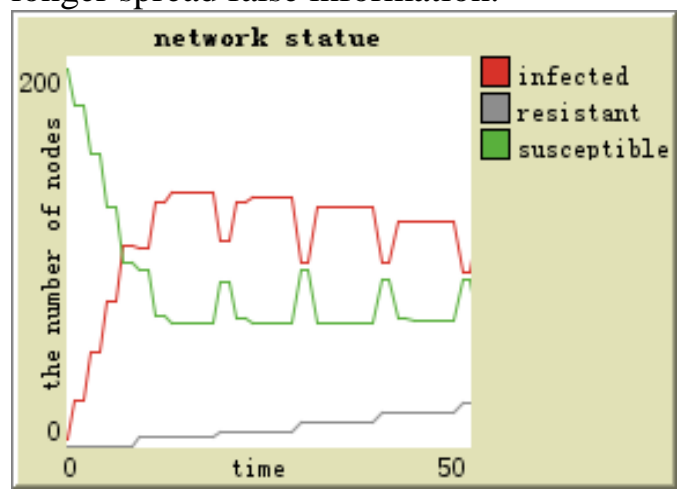

Figure 3. The Value of Initial Infected Nodes is 1

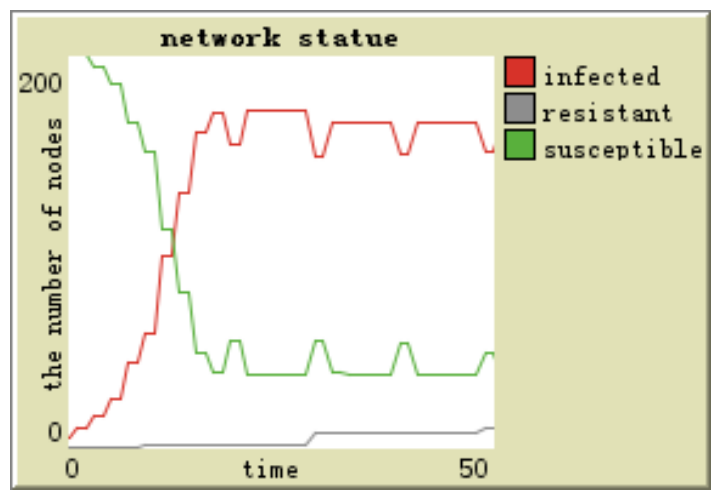

Figure 4. The Value of Initial Infected Nodes is 3

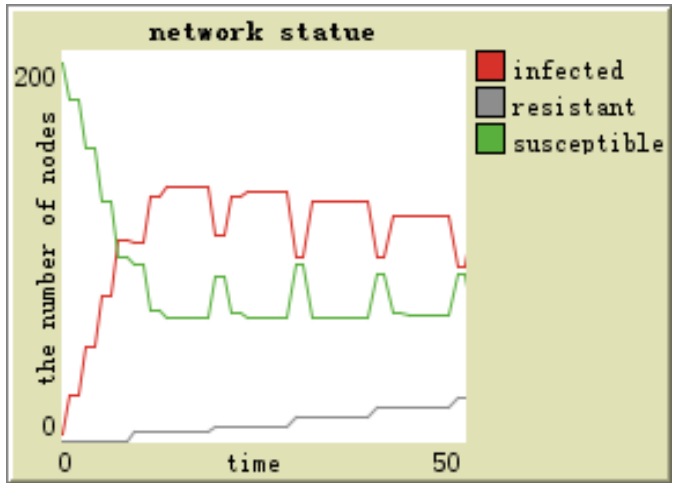

Figure 5. The Value of Initial Infected Nodes is 6

The Figure 3, 4, 5 show that when the value of initial infected nodes is 1 , the largest outbreak scale appears in the simulation time 17-18, and the value is 164; when the value of initial infected nodes is 3 , the largest outbreak scale appears in the simulation time 1318 , and the value is 166 ; when the value of initial infected nodes is 6 , the largest outbreak scale appears in the simulation time 15-18, and the value is 107.

The fastest to reach the largest outbreak scale is when the value of initial infected nodes is 3 , and it maintains the longest time.

In $B_{1}$, the largest outbreak scale accounted for $83 \%$ of total scale which is more than $A_{1}$ by $1 \%$, and which is more than $C_{1}$ by $29.5 \%$. To maintain the size of the largest outbreak time account for $10 \%$ of the total simulation time which is more than $A_{1}$ by $8 \%$, and which is more than $C_{1}$ by $4 \%$.

When public crisis false information occurs, it is the individuals in the personal networks whose number of edges is in the intermediate state. They have wider range of spread and spread Enthusiasm obviously. These individuals are active in the social life, they are not a leader, but they have a certain appeal. When public crisis event occurs, they always show great enthusiasm. But they understand the state of the event with their subjective color, and with a certain degree of blindness. 


\section{The Simulation Experimental Designs and Results Analysis of False Information Diffusion in Public Crisis under Different Intervention Conditions}

From the intervention strategic point of view as designed in Figure 6, when the public crisis false information generates and diffuses, $C_{2}, C_{3}, C_{4}$ program are executed and we get the following results.

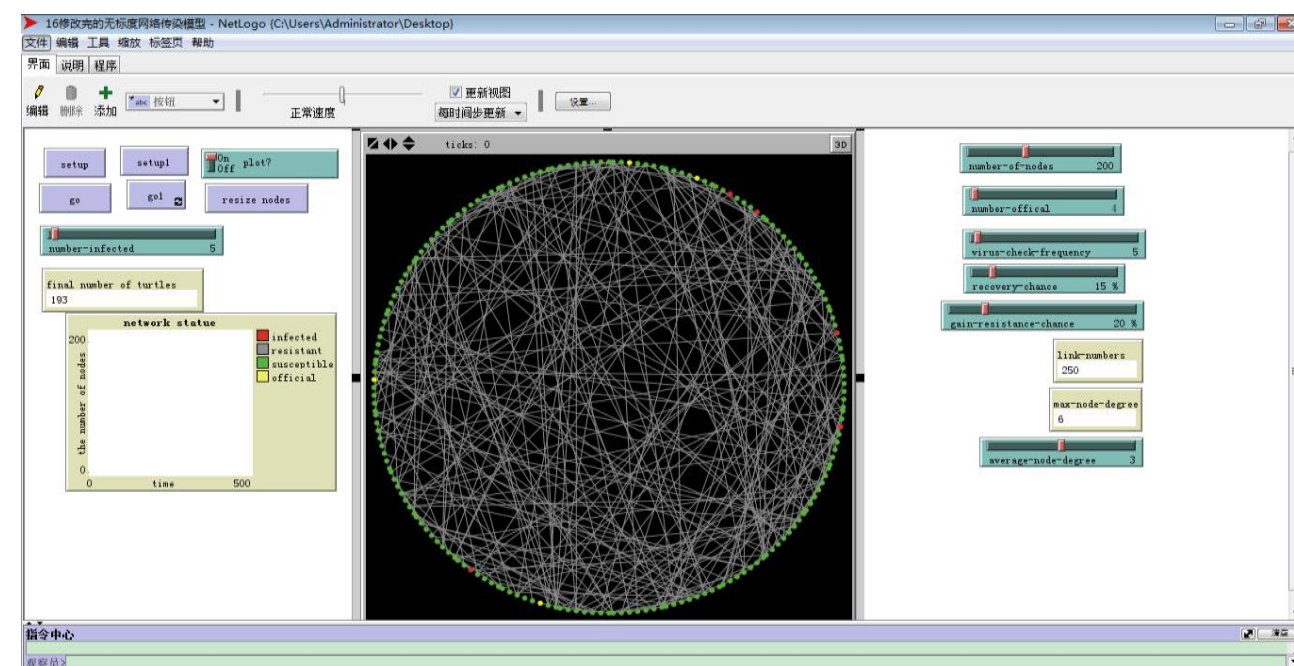

\section{Figure 6. Experiment Design of False Information Diffuse under Different Intervention Conditions}

On the basis of the network model of diffusing freely, intervention nodes are added through go1 routine and network statue of drawing program is edited, drawing the number of four states individuals graphs: infected (information recipients), susceptible (easy to accept, but those who have not accepted the message), resistant (who are not interested in information and stop spreading), official (the number of the individual who receive the official message). The results are as follows:

(1) As is shown in figure $7,8,9, C_{2}, C_{3}, C_{4}$ programs are respectively carried out to intervene the progress of false information diffusion. First intervention nodes are added. Setting the nodes with 6 links initial value $0,0.5,1$, which respectively represents information views were opposed, neutral and supportive. Simulation results show that when the initial attitude of the intervention node is 0 (that is opposition, the program $C_{2}$ is carried out) the maximum number of infected nodes are more $18 \%$ than the number of the infected node when the initial attitude of the intervention node is 1 (that is acceptance the false information, the program $C_{4}$ is carried out). When the initial attitude of the intervention is 0.5 (that is neutral, the program $C_{4}$ is carried out), the maximum number of infected nodes (receiving the false information) is less 13\% than the number of the infected node when the initial attitude of the intervention node is 1 (that is acceptance the false information, the program $C_{4}$ is carried out). When the initial attitude of the intervention is 0.5 (that is neutral, the program $C_{4}$ is carried out), the maximum number of infected nodes (receiving the false information) is less $20 \%$ than the number of the infected node when the initial attitude of the intervention node is 0 (that is opposition, the program $C_{2}$ is carried out). 


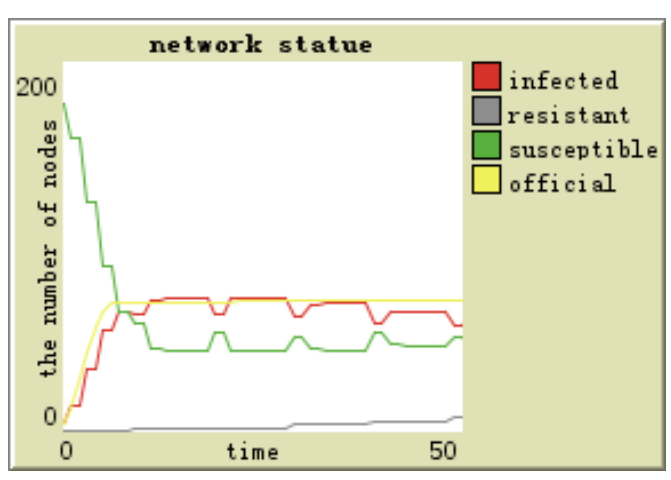

Figure 7. The Official Information is 0

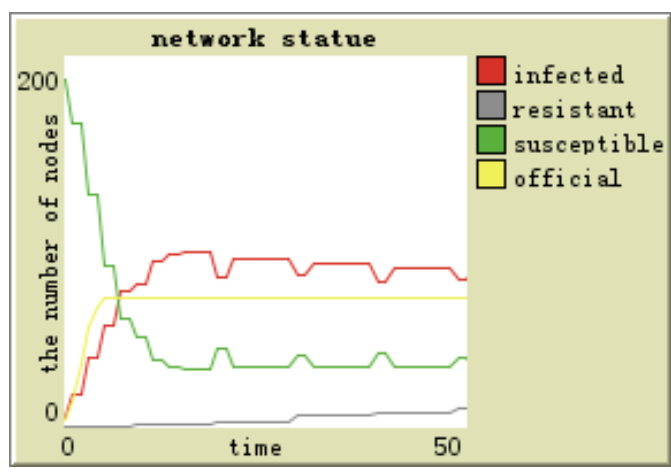

Figure 8. The Official Information is 1

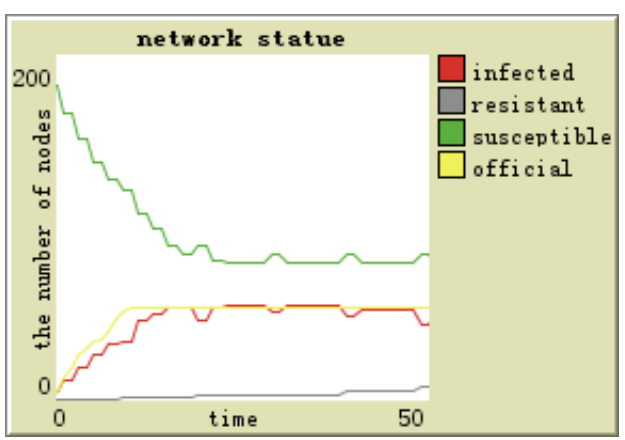

Figure 9. The Official Information is $\mathbf{0 . 5}$

(2) As is shown in Figure $10,11,12, A_{3}, B_{3}, C_{3}$ programs are respectively carried out to intervene the progress of false information diffusion., the nodes with 1, 3, 6 links are selected as intervention nodes, and the neutral information (0.5) are released though intervention nodes. When the $C_{3}$ program is carried out, that is the intervention is carried on the node with 6 links (the number of edges is 6 , communication range are in high level) the maximum number of infected nodes (receiving the false information) is respectively less $23 \%$ and $12 \%$ than ${ }^{A_{3}}$ program (the intervention is carried on the node with 1 links, the number of edges is 1 , communication range are in low level) and ${ }^{B_{3}}$ program(the intervention is carried on the node with 3links, the number of edges is 3, communication range are in medium level). The $C_{3}$ program is the most effective in reducing the number of receiving false information in public crisis, increasing the number of individuals affected by the public media. 


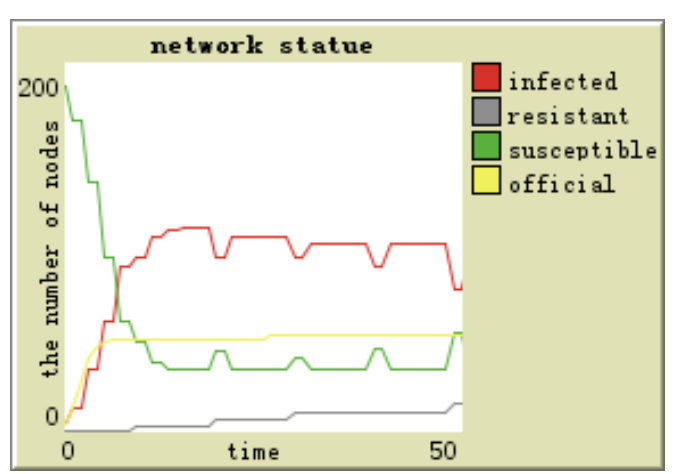

Figure 10. The Degree of Official is 1

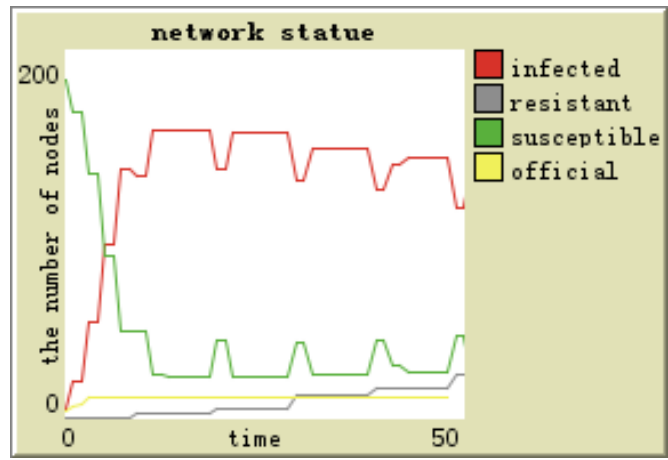

Figure 11. The Degree of Official is 3

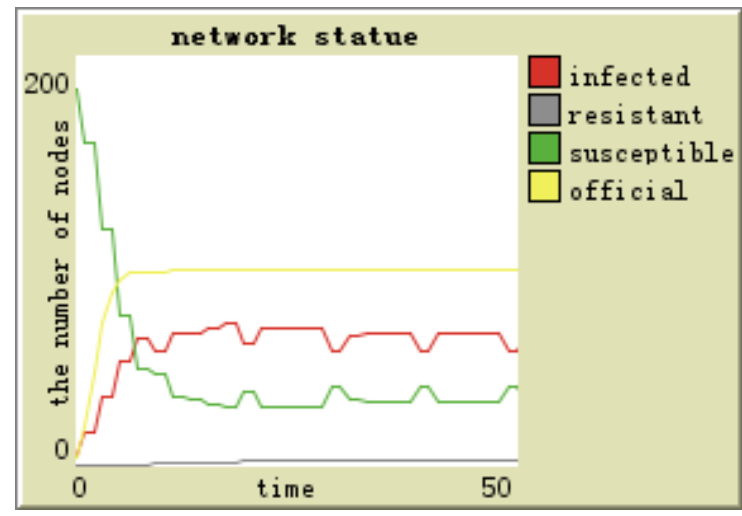

Figure 12. The Degree of Official is 6

\section{Conclusion}

1. When false information in public crisis diffusion without any intervention, the time of final burst will be longer or the speed will be slower if the initial false information receiver has a wide range of communication scope. Besides, the final burst size will be smaller. Instead, when the initial recipients of false information is the scope of general individual, the diffusion speed will be faster and the final burst size will be larger. While when the range of initial recipients of false information is small, the diffusion speed will be slower. However, the change of final burst size is not obvious. This conclusion disobeys that people with large communication range have stronger communication diffusion ability. Instead, people with the range of intermediate state in the communication network have stronger communication diffusion ability.

2. When the false information in public crisis diffuses, the management department of false information in public crisis should not publish the completely contradict information with the diffusion information. That is to say, they should not take confrontational intervention measures, or it will excite the public to produce stronger negative information. At this time, the management department of false information in public crisis should maintain a circuitous neutral attitude rather than the "all-or nothing" coercive measures when intervene the diffusion process of the false information diffusion.

3. The leading position node plays an important role in the process of the diffusion of false information. When false information managers not intervene, the main spread subjects are the individuals of the middle level in the communication range. The se individuals are active in the diffusion of false information. However, from the perspective of intervention scope, when the false information management makes intervention to the diffusion of false information, the key object is the leading position node. Therefore, when the false information in public crisis diffuses, the key control objects are the public 
with a wide range of communication scope, because it can control the development of situation quickly. However, the intermediate state node should not be ignored.

\section{Acknowledgements}

This research is supported in part by the Study of diffusion mechanism and control of false information in public crisis of National Natural Science Fund (71301140); Natural Science Fund of Hebei Province (G2015203425); Hebei higher school science and technology research project "mechanism and evolution of false information intervention study in public emergency" (QN20131090).

\section{References}

[1] Jar-der Luo. Social Network Analysis Notes, social sciences academic press, Beijing, (2005).

[2] A. Rapoport and L. Rebhun, "On the mathematical theory of rumor spread", Bulletin of Mathematical Biology, vol. 4, (1952), pp. 375-383.

[3] B. W. Goffman and V. A. Newill, "Communication and epidemic processes", Nature, vol. 204, no. 225, (1964).

[4] D. J. Daley and D. G. Kendal, Stochastic rumours. Inst. Maths Applies, vol. 42, no. 55, (1965).

[5] Z. Jian, M. Zhien and H. Maoan, Global Stability of an SIRS Epidemic Model with Delays Acta Mathematics Scientia, 26B, no. 2, (2006), pp. 291-306.

[6] X. Lanfang and X. Aimin and Fan Xiaofeng, "Computer network virus propagation model SIRH [J]", Computer Engineering and Science, vol. 31, no. 1, (2009), pp. 4-6.

[7] Maki D. and Thomson M., "Mathematical Models and Applications [M]", New Jersey: Prentice-Hall, Englewood Cliff, (1973).

[8] Murray J. D., "Mathematical Modeling in Epidemiology [M]", Berlin: Springer, (1980).

[9] R. E. Dickinson and C. E. M. Pearce, "Rumours, Epidemics, and Processes of Mass Action: Synthesis and Analysis", Mathematical and Computer Modeling, no. 38, (2003), pp. 1157-1167.

[10] K. A. Thompson, "Deterministic Approach to the Spread of Rumors", Technical Report BU-1642-M, (2003).

[11] K. Kawachi, "Deterministic models for rumor transmission", Nonlinear Analysis: Real World Applications, (2008) September, pp. 1989 -2028.

[12] K. Kawachi and M. Seki, "A rumor transmission model with various contact interactions", Journal of Theoretical Biology, vol. 253, (2008), pp. 55-60.

[13] L. A. Huo, P. Q. Huang and X. Fang, "An interplay model for authorities' actions and rumor spreading in emergency event”, Physical A: Statistical Mechanics and its Applications, vol. 20, no. 1, (2011), pp. 3267-3274.

[14] zhongqi, qiwei, zhangle, "Social type crisis information diffusion model under [J]", Systems Engineering Theory and Practice, vol. 3201, (2012), pp. 104-110.

[15] Zanette, "Dynamics of Rumor Propagation on Small - world Networks[J]", American Physical Society, (2002), pp. 8- 9.

[16] M. W. Seeger, T. L. Sellnow and R. R. Ulmer, "Communications, Organization and Crisis [M]", Thousand Oaks, CA: Sage Publications, (1998).

[17] C. L. Barrett, S. G. Eubank and J. P. Smith, "If small pox strikes Port land [J]", Scientific American, vol. 292, no. 3, (2005), pp. 42-49.

[18] Monge P. R. and Noshir C., "Theories of Communication Networks [M]", NY: Oxford University Press. (2003).

[19] KrauseU., "A discrete nonlinear and non-autonomous model of consensus formation [A]", Communications in Difference Equations [C], Amsterdam: Gordon and Breach Pub., (2000), pp. 227236. 


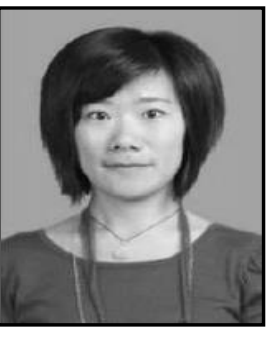

Zhu Xiaoxia, she received her Ph.D. degree in Management Science and Engineering from Haerbin Engineering University, China in 2008. She is currently an Associate Professor in the Economics and Management School at Yanshan University, China. She also works for the Ministry of Industry and Information Technology as an Economic Analyst. Her main research interests include complex networks, information dissemination, and data mining.

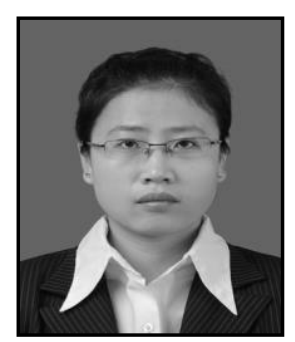

Hao jiajia, he is pursuing a doctor's degree in Management Science and Engineering in Yanshan University. 
International Journal of Security and Its Applications

Vol. 10, No. 10 (2016) 